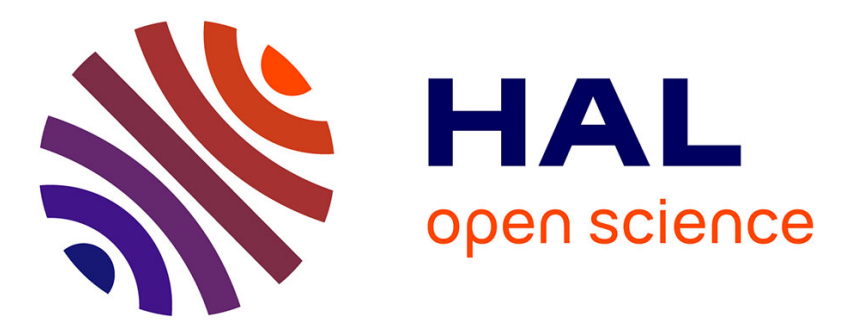

\title{
Integer and Floating-Point Constant Multipliers for FPGAs
}

Nicolas Brisebarre, Florent de Dinechin, Jean-Michel Muller

\section{To cite this version:}

Nicolas Brisebarre, Florent de Dinechin, Jean-Michel Muller. Integer and Floating-Point Constant Multipliers for FPGAs. International Conference on Application-Specific Systems, Architectures and Processors, 2008, IMEC, Jul 2008, Leuven, Belgium. pp.239-244, 10.1109/ASAP.2008.4580184 ensl00269219

\section{HAL Id: ensl-00269219 \\ https://hal-ens-lyon.archives-ouvertes.fr/ensl-00269219}

Submitted on 2 Apr 2008

HAL is a multi-disciplinary open access archive for the deposit and dissemination of scientific research documents, whether they are published or not. The documents may come from teaching and research institutions in France or abroad, or from public or private research centers.
L'archive ouverte pluridisciplinaire HAL, est destinée au dépôt et à la diffusion de documents scientifiques de niveau recherche, publiés ou non, émanant des établissements d'enseignement et de recherche français ou étrangers, des laboratoires publics ou privés. 


\title{
Integer and Floating-Point Constant Multipliers for FPGAs
}

\author{
Nicolas Brisebarre, Florent de Dinechin, Jean-Michel Muller* \\ LIP (CNRS/INRIA/ENS-Lyon/UCBL) \\ Université de Lyon \\ \{Nicolas.Brisebarre, Florent.de.Dinechin, Jean-Michel.Muller\}@ens-lyon.fr
}

\begin{abstract}
Reconfigurable circuits now have a capacity that allows them to be used as floating-point accelerators. They offer massive parallelism, but also the opportunity to design optimised floating-point hardware operators not available in microprocessors. Multiplication by a constant is an important example of such an operator. This article presents an architecture generator for the correctly rounded multiplication of a floating-point number by a constant. This constant can be a floating-point value, but also an arbitrary irrational number. The multiplication of the significands is an instance of the well-studied problem of constant integer multiplication, for which improvement to existing algorithms are also proposed and evaluated.
\end{abstract}

\section{Introduction}

FPGAs (for field-programmable gate arrays) are highdensity VLSI chips which can be programmed to efficiently emulate arbitrary logic circuits. Where a microprocessor is programmed at the granularity of instructions operating on 32 or 64-bit data words, FPGAs are programmed at the bit and register level. This finer grain comes at a cost: a circuit implemented in an FPGA is typically ten times slower than same circuit implemented as an ASIC (application-specific integrated circuit). Despite this intrinsic performance gap between FPGAs and ASIC, the former are often used as a replacement of the latter for applications which don't justify the non-recurring costs of an ASIC, or which have to adapt to evolving standards.

FPGAs have also been used as configurable accelerators in computing systems. They typically excel in computations which exhibit massive parallelism and require operations absent from the processor's instruction set.

\footnotetext{
${ }^{*}$ This work was partly supported by the XtremeData university programme, the ANR EVAFlo project and the Egide Brâncuşi programme 14914RL.
}

The FloPoCo project aims at exploring the implementation of such non standard operations, especially in the floating-point realm [4]. This article is a survey of the issue of multiplication by a constant in this context.

\section{State of the art and contributions}

Multiplication by a constant is a pervasive operation. It often occurs in scientific computating codes, and is at the core of many signal-processing filters. It is also useful to build larger operators: previously published architectures for exponential, logarithm and trigonometric functions $[8,7]$ all involve multiplication by a constant. A single unoptimised multiplication by $4 / \pi$ may account for about one third the area of a dual sine/cosine operator [7].

The present article essentially reconciles two research directions that were so far treated separately: on the one side, the optimisation of multiplication by an integer constant, addressed in section 2, and on the other side the issue of correct rounding of multiplication or division by an arbitrary precision constant, addressed in section 4 .

Integer constant multiplication has been well studied, with many good heuristics published [3, 6, 13, 5, 1, 15]. Its theoretical complexity is still an open question: it was only recently proven sub-linear, although using an approach which is useless in practice $[9,15]$. Our contribution in this domain is essentially a refinement of the objective function: where all previous works to our knowledge try to minimise the number of additions, we remark that these additions, measured in terms of full adder cells, have different sizes (up to a factor 4 for the large multiplier by $4 / \pi$ of [7]), hence variable cost in reconfigurable logic. Trying to minimise the number of full adders, and looking for low-latency and easy to pipeline architectures, we suggest a surprisingly simple algorithm that, for constants up to 64 bits, outperforms the best known algorithms in terms of FPGA area usage and latency. Boullis and Tisserand [1] also tried to minimise adder size, but as a post-processing step, after an algorithm minimising the number of additions. 
Section 3 describes a multiplier by a floating-point constant of arbitrary size. The architecture is a straightforward specialisation of the usual floating-point multiplier. It is actually slightly simpler, because the normalisation of the result can be removed from the critical path.

Finally, Section 4 deals with the correct rounding of the multiplication by an arbitrary real constant. Previous work on the subject [2] has shown that this correct rounding requires a floating-point approximation of the constant whose typical size is twice the mantissa size of the input. This size actually depends on the real constant, and may be computed using a simple continued fractions algorithm. The other contribution of [2] is the proof of an algorithm which consists of two dependent fused-multiplyand-add operations. In the FPGA, the implementation will be much simpler, since it will suffice to instantiate a large enough FP constant multiplier. Of course, a multiplier by an arbitrary constant is also capable of computing the division by an arbitrary constant [14].

What the previous means is that the price of correct rounding, for a multiplication by an irrational constant like $\pi$ or $\log 2$, will be a typical doubling of the number of bits of the constant that have to be used in significand multiplication. As the cost of such a multiplication is sublinear in the constant size [9], the price of correct rounding is actually less than this factor 2 in practice.

All these architectures are implemented in the FloPoCo framework.

\section{Multiplication by an integer con- stant}

Several recent papers $[1,9,15]$ will provide the interested reader with a state of the art on this subject.

Let $C$ be a positive integer constant, written in binary on $k$ bits:

$$
C=\sum_{i=0}^{k} c_{i} 2^{i} \quad \text { with } c_{i} \in\{0,1\}
$$

Let $X$ a $p$-bit integer. The product is written $C X=$ $\sum_{i=0}^{k} 2^{i} c_{i} X$, and by only considering the non-zero $c_{i}$, it is expressed as a sum of $2^{i} X$. For instance, $17 X=X+$ $2^{4} X$. In the following, we will note this using the shift operator $\ll$, which has higher priority than + and - . For instance $17 X=X+X \ll 4$.

If we allow the digits of the constant to be negative $\left(c_{i} \in\{-1,0,1\}\right)$ we obtain a redundant representation, for instance $15=01111=1000 \overline{1}(16-1$ written in signed binary). Among the representations of a given con- stant $C$, we will pick up one that minimises the number of non-zero bits, hence of additions/subtractions.

The well-known canonical signed digits recoding (or CSD, also called Booth recoding [10]) garantees that at most $k / 2$ bits are non-zero, and in average $k / 3$.

\subsection{Parenthesing and architectures}

The CSD recoding of a constant may be translated into a rectangular architecture[5], an example of which is given by Figure 1. This architecture corresponds to the following parenthesing: $221 X=X \ll 8+(-X \ll 5+(-X \ll$ $2+X))$

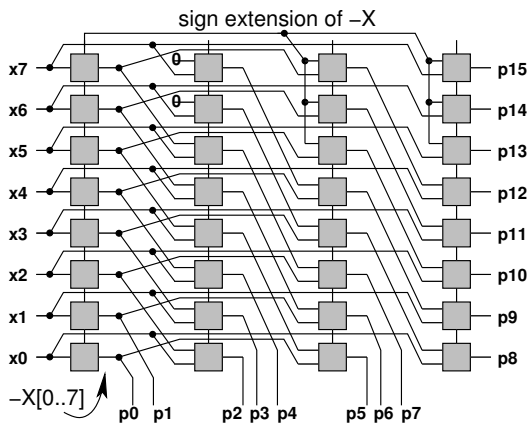

Figure 1: Multiplier of an 8-bit input by 221, using the recoding $100 \overline{1} 00 \overline{1} 01$

We introduce a new tree adder structure that is constructed out of the CSD recoding of the constant as follows: non-zero bits are first grouped by 2 , then by 4 , etc. For instance, $221 X=(X \ll 8-X \ll 5)+(-X \ll 2+X)$. This new way of parenthesing the sum reduces the critical path: For $k$ non-zero bits, it is now of $\left\lceil\log _{2} k\right\rceil$ additions instead of $k$ in the linear architecture of Figure 1.

Besides, shifts may also be reparenthesised: $221 X=$ $(X \ll 3-X) \ll 5+(-X \ll 2+X)$. After doing this, the leaves of the tree are now multiplications by small constants: $3 X, 5 X, 7 X, 9 X \ldots$ Such a smaller constants will appear many times in a larger constant, but it will have to be computed only once: the tree now becomes a DAG (direct acyclic graph), and the number of additions is reduced. A larger example is shown on Figure 2.

\subsection{Lefèvre's constant multipliers}

We have saved adders by going from a tree to a DAG. Lefèvre [13] has generalised this idea to an algorithm that minimises the number of adders: it looks for maximal repeating bit patterns in the CSD representation, and generates them recursively. Lefèvre observed that the number 
Of course, architecture generation will produce hardware only for the useful parts of the adders (see also Figure 1). Space is missing to exhibit VHDL produced by FloPoCo, but readers are invited to try it out.

This cost function describes relatively acurately the cost of a combinatorial constant multiplier. It has to be extended to the case of pipelined multipliers: one has to add the overhead of the registers, essentially for the lower bits since a registered adder has the same cost as a combinatorial one in FPGAs. In principle, one pipeline stage may contain several DAG levels, at least for the lower levels.

\subsection{The IntConstMult class in FloPoCo}

FloPoCo currently implements this DAG structure and outputs VHDL for it. It also implements cost analysis, but no design space exploration based on it: it currently only builds the simple DAGs illustrated by Figure 1 and Figure 2.

For comparison, two sequences produced by rigo . C, for the significands of $\pi / 2 \times 2^{50}$ and $\pi / 2 \times 2^{107}$, were hand-translated into FloPoCo DAGs. From the synthesis results of Table 1 (these are FP multipliers, but their area and delay are largely dominated by the significant multiplication), one observes that for the 50-bit constant, although the number of additions is smaller, the final area is larger, as many of these additions are very large. This justifies the introduction of a new cost function. More work is needed to actually use it in an optimisation program. Fair comparison would also require to apply to the DAG given by rigo. c post-optimisations suggested by [1].

\section{Multiplication by a floating-point constant}

For the needs of this article, an FP number is written $(-1)^{s} \cdot 2^{E} \cdot 1, F$ where $1, F \in[1,2[$ is a significand and $E$ is a signed exponent. We shall note $w_{E}$ and $w_{F}$ the respective sizes of $E$ and $F$, and $\mathbb{F}\left(w_{E}, w_{F}\right)$ the set of FP numbers in a format defined by $\left(w_{E}, w_{F}\right)$. We want to allow for different values of $w_{E}$ and $w_{F}$ for the input $X$ and the output $R$ :

$$
\begin{array}{ll}
X=(-1)^{s_{X}} \cdot 2^{E_{X}} \cdot 1, F_{X} & \in \mathbb{F}\left(w_{E_{X}}, w_{F_{X}}\right) \\
R=(-1)^{s_{R}} \cdot 2^{E_{R}} \cdot 1, F_{R} & \in \mathbb{F}\left(w_{E_{R}}, w_{F_{R}}\right)
\end{array}
$$

In all the following, the real value of the constant will be noted $C$, possibly an irrational number, and we define

$$
C=(-1)^{s_{C}} \cdot 2^{E_{C}} \cdot 1, F_{C}
$$

the unique floating-point ${ }^{2}$ representation of $C$ such that $1, F_{C} \in\left[1,2\left[\right.\right.$. Here $F_{C}$ may have an infinite binary representation. We note $C_{k}$ the approximation of $C$ rounded to the nearest on $w_{F_{C}}=k$ fraction bits:

$$
C_{k}=(-1)^{s_{C}} \cdot 2^{E_{C}} \cdot 1, F_{C_{k}}
$$

Finally, we also define the real number

$$
1, F_{\text {cut }}=\frac{2}{1, F_{C}} \quad \in[1,2[
$$

We now describe a multiplier that computes the correct rounding $R_{k}$ of $C_{k} \times X$. Then, Section 4 will compute the minimal $k$ ensuring that $\forall X \in \mathbb{F}\left(w_{E_{X}}, w_{F_{X}}\right), R_{k}$ is the correct rounding of $C \times X$.

Of course, if $C$ is already a $p$-bit-significand FP number, it will be $k=p$.

The architecture given by Figure 3, and implemented as the FPConstMult class in FloPoCo, is essentially a simplification of the standard FP multiplier. The main modification is that rounding is simpler. In the standard multiplier, the product of two significands, each in $[1,2)$, belongs to $[1,4)$. Its normalisation and rounding is decided by looking at the product. In a constant multiplier, it is possible to predict if the result will be larger or smaller than 2 just by comparing $F_{X}$ with $F_{c u t}$ - in practice, with $F_{c u t}$ truncated to $w_{F_{X}}$ bits. This is also slightly faster, as the rounding decision is moved off the critical path.

Exponent computation consists in adding the constant exponent, possibly augmented by 1 if $F_{X}>F_{\text {cut }}$. Sign computation is also straightforward. Exceptional case handling is also slighly simpler. For instance, if the constant has a negative exponent, one knows that an overflow will never occur. Likewise, if it is positive or zero, underflow (flush to zero) cannot happen.

\section{Correct rounding of the multipli- cation by a real constant}

This section proposes a method for computing the minimal value of $k=w_{F_{C}}$ allowing for correct rounding (noted $\circ$ ) of the product of any input $X$ by $C$. First, for a given $k$, we show how to build a predicate telling if there exist values of $X$ such that $R_{k}=\circ\left(C_{k} X\right) \neq \circ(C X)$. This allows us to look for the minimal $k$ verifying this predicate, knowing that it is generally expected to be close to $2 w_{F_{X}}$.

\footnotetext{
${ }^{2}$ As the exponent is constant, the point doesn't actually float at all.
} 


\begin{tabular}{|c||c|c|c||c|c|c||c|c|c|}
\hline \multicolumn{1}{|c||}{} & \multicolumn{3}{c||}{ FloPoCo linear CSD } & \multicolumn{3}{c||}{ FloPoCo DAG CSD } & \multicolumn{3}{c|}{ Lefèvre/Rigo } \\
\hline constant & + & LUTs & delay & + & LUTs & delay & + & LUTs & delay \\
\hline \hline$X$ on 24 bits, $\pi / 2$ on 50 bits & 19 & 435 & $30 \mathrm{~ns}$ & 15 & 467 & $14 \mathrm{~ns}$ & 12 & 645 & $16 \mathrm{~ns}$ \\
\hline$X$ on 53 bits, $\pi / 2$ on 107 bits & 38 & 2018 & $68 \mathrm{~ns}$ & 26 & 1628 & $21 \mathrm{~ns}$ & 22 & 1508 & $18 \mathrm{~ns}$ \\
\hline
\end{tabular}

Table 1: Synthesis results for floating-point multipliers

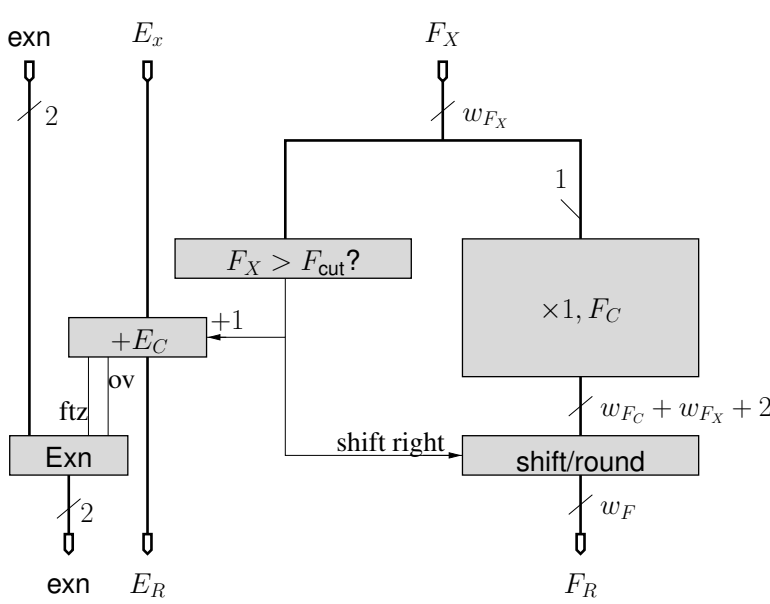

Figure 3: Multiplier by an FP constant

\subsection{Existence of an $X$ such that $\circ\left(C_{k} X\right) \neq$ $\circ(C X)$}

The FP multiplier guarantees the correct rounding of the result of the multiplication by $C_{k}$, that is to say,

$$
\begin{aligned}
\forall X \in \mathbb{F}\left(w_{E_{X}}, w_{F_{X}}\right), & \left|R_{k}-C_{k} X\right| \\
\leq & \frac{1}{2} \operatorname{ulp}\left(C_{k} X\right) \leq \frac{1}{2} \operatorname{ulp}\left(R_{k}\right),
\end{aligned}
$$

in which "ulp(t)" (unit in the last place) is the weight of the least significant bit of $t$.

Moreover, $C_{k}$ is also the rounded-to-nearest value of $C$. Let $\varepsilon_{a}=\left|C_{k}-C\right|$, we have

$\forall X \in \mathbb{F}\left(w_{E_{X}}, w_{F_{X}}\right), \quad\left|R_{k}-C X\right| \leq \frac{1}{2} \operatorname{ulp}\left(R_{k}\right)+X \cdot \varepsilon_{a}$.

We may assume, without any loss of generality, that $X$ and $C$ belong to $[1,2)$, i.e. $E_{X}=E_{C}=0$. Then we have

$$
\begin{aligned}
\forall X \in \mathbb{F}\left(w_{E_{X}}, w_{F_{X}}\right), 1 \leq X & <2, \\
\left|R_{k}-C X\right| & <\frac{1}{2} \operatorname{ulp}\left(R_{k}\right)+2 \varepsilon_{a}
\end{aligned}
$$

If we can prove that for all $X,\left|R_{k}-C X\right| \leq \frac{1}{2}$ ulp $\left(R_{k}\right)$, then $R_{k}$ will always be the closest FP number to $C X$,

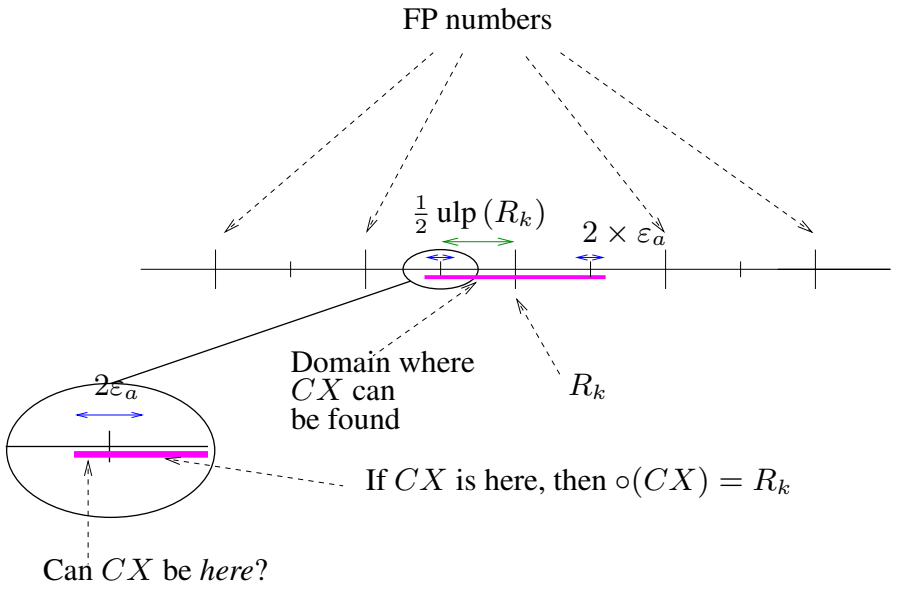

Figure 4: If $C X$ is at a distance greater than $1 / 2$ ulp of $R_{k}$, then it is at a distance lesser than $2 \varepsilon_{a}$ from the middle of two consecutive FP numbers.

which is the required property. As shown in Figure 4.1, if $C X$ satisfies to (1) and is at a distance greater than $\frac{1}{2} \mathrm{ulp}\left(R_{k}\right)$ from $R_{k}$, it is necessarily at a distance lesser than $2 \varepsilon_{a}$ from the middle of two consecutive FP numbers. Such a point is a rational number of the form $(2 A+1) /(2 q)$, with $2^{w_{F_{R}}} \leq A \leq 2^{w_{F_{R}}+1}-1$ and $q=2^{w_{F_{R}}+t}$, where $t$ is equal to 1 if $C X$ has the same exponent as $X$ (if $F_{X} \leq F_{c u t}$ ), and is equal to 0 otherwise.

Therefore, to determine if an input $X$ is such that $R_{k}$ is not the correct rounding of $C X$, one can check first if there exists an approximation to $C X$ by a rational number of the form $(2 A+1) /(2 q)$, such that $\mid C X-(2 A+$ 1) $/(2 q) \mid \leq 2 \varepsilon_{a}$.

The mathematical tool for solving this kind of rational approximation issues is continued fractions [11]. Using them, one can design several methods [2] that make it possible either to guarantee that $C X$ will not be at a distance lesser than $2 \varepsilon_{a}$ from the middle of two consecutive FP numbers (hence one can guarantee that the correct rounding of CX is always returned) or to compute all counter-examples, that is to say values of $X$ such that 
$C_{k} X$ rounded to nearest is not the correct rounding of $C X$. In the latter case, one can derive from each counterexample the value by which we should increment $k$ in order to get a correct rounding.

\subsection{A predicate for $k$}

We assume in the sequel that $F_{X}<F_{c u t}$ (the case $F_{X}>$ $F_{\text {cut }}$ is similar). We then have $C X \in[1,2)$. Let $M_{X}$ be the integer mantissa of $X$, i.e. $M_{X}=2^{w_{F_{X}}} X$. We search for the integers $M_{X} \in \mathbb{Z}$ such that

$$
\left|\frac{M_{X}}{2^{w_{F_{X}}}} C-\frac{2 A+1}{2^{w_{F_{R}}}+1}\right| \leq 2 \varepsilon_{a} .
$$

Depending on the relative values of $w_{F_{R}}$ and $w_{F_{X}}$, we face two situations:

\subsubsection{Case where $w_{F_{R}}+1 \geq w_{F_{X}}$}

We assume in this case that $k=w_{F_{R}}+w_{F_{X}}+3$. We search for the integers $M_{X} \in \mathbb{Z}$ such that

$$
\left|M_{X} 2^{w_{F_{R}}-w_{F_{X}}+1} C-2 A-1\right| \leq 2^{2+w_{F_{R}}} \varepsilon_{a} .
$$

Since $\varepsilon_{a}=\left|C_{k}-C\right| \leq 2^{-k-1}$, we have

$$
\left|M_{X} 2^{w_{F_{R}}-w_{F_{X}}+1} C-2 A-1\right| \leq 2^{w_{F_{R}}-k+1} .
$$

Note that $2^{w_{F_{R}}-k+1}<1 /\left(2 M_{X}\right)$ iff $2^{w_{F_{R}}-k+2} M_{X}<$ 1. As $M_{X}<2^{w_{F_{X}}+1}$ and $2^{w_{F_{R}}+w_{F_{X}}-k+3} \leq 1$ since we assumed $w_{F_{R}}+w_{F_{X}}+3=k$, we have $2^{w_{F_{R}}-k+1}<$ $1 /\left(2 M_{X}\right)$ for all $X \in[1,2[:$ we compute the continued fraction expansion of $2^{w_{F_{R}}-w_{F_{X}}+1} C$ that yields (all the) candidate values $X$ that may possibly satisfy $\circ(C X) \neq$ $\circ\left(C_{k} X\right)$. For all such input $X$, we first check exhaustively if those rounded values actually differ and we collect all such $X$ in a list $L$. Then, we compute the minimal value $\eta$ of $\left|\frac{M_{X}}{2^{w} F_{X}} C-\frac{2 A+1}{2^{w} F_{R}+1}\right|$ when $X$ ranges the list $L$ of all counter-examples and we set $k=\max \left(w_{F_{R}}+w_{F_{X}}+\right.$ $\left.3,\left\lceil-\log _{2}(\eta)\right\rceil+1\right)$. The inequality $k \geq\left\lceil-\log _{2}(\eta)\right\rceil+$ 1 imples $k>-\log _{2}(\eta)$ that yields $2 \varepsilon_{a} \leq 2^{-k}<\eta$, which guarantees that all inputs $X$ will satisfy $\circ(C X)=$ $\circ\left(C_{k} X\right)$.

\subsubsection{Case where $w_{F_{R}}+2 \leq w_{F_{X}}$}

We assume in this case that $k=2 w_{F_{X}}+2$. We search for the integers $M_{X} \in \mathbb{Z}$ such that

$$
\left|M_{X} C-(2 A+1) 2^{w_{F_{X}}-w_{F_{R}}-1}\right| \leq 2^{1+w_{F_{X}}} \varepsilon_{a} .
$$

Here, again, we use $\varepsilon_{a}=\left|C_{k}-C\right| \leq 2^{-k-1}$ to infer

$$
\left|M_{X} C-(2 A+1) 2^{w_{F_{X}}-w_{F_{R}}-1}\right| \leq 2^{w_{F_{X}}-k} .
$$

\begin{tabular}{|c||c|c|c|}
\hline mult. by $\pi / 2, w_{F_{X}}=53$ & + & LUTs & delay \\
\hline \hline standard $\left(w_{F_{C}}=53\right)$ & 16 & 866 & $20 \mathrm{~ns}$ \\
correct rounding $\left(w_{F_{C}}=107\right)$ & 26 & 1628 & $21 \mathrm{~ns}$ \\
\hline
\end{tabular}

Table 2: The price of correct rounding

Here again, from the hypothesis $2 w_{F_{X}}+2 \leq k$, we infer $2^{w_{F_{X}}-k}<1 /\left(2 M_{X}\right)$ : the computation of the continued fraction expansion of $C$ provides a (complete) list of values $X$ candidate for satisfying $\circ(C X) \neq \circ\left(C_{k} X\right)$. We check exhaustively if those rounded values actually differ and we collect again all such $X$ in a list $L$. Let $\eta$ be the minimal value of $\left|\frac{M_{X}}{2^{w_{F}} F_{X}} C-\frac{2 A+1}{2^{w_{F}} F_{R}+1}\right|$ when $X$ ranges the list $L$ of all counter-examples. We set $k=\max \left(w_{F_{R}}+w_{F_{X}}+3,\left\lceil-\log _{2}(\eta)\right\rceil+1\right)$. That value of $k$ will ensure that $\circ(C X)=\circ\left(C_{k} X\right)$ for all input $X$.

These computations are currently performed in Maple, but will soon be integrated in the constant multiplier generator.

\section{Conclusion and perspectives}

One may argue that floating-point multiplication is too anecdotical to justify so much effort. Yet it illustrates what we believe is the future of floating-point on FPGAs: thanks to their flexibility, they may accomodate non-standard optimised operators, for example a correctly rounded multiplication by an irrational constant. It also makes a good case study for the implementation of such non-standard operators: they cannot be offered as offthe-shelf libraries, they have to be optimised for each application-specific context. This is the object of the FloPoCo project. Near-term future work will focus on automatically pipelining these operators for a given target frequency. Our results also suggest that there is much room for improvement in the optimisation of integer multiplication: we have defined a pertinent design space, but the exploration of this space is yet to implement.

\section{References}

[1] N. Boullis and A. Tisserand. Some optimizations of hardware multiplication by constant matrices. IEEE Transactions on Computers, 54(10):1271-1282, Oct. 2005.

[2] N. Brisebarre and J.-M. Muller. Correctly rounded multiplication by arbitrary precision constants. IEEE Transactions on Computers, 57(2):165-174, feb 2008. 
[3] K. Chapman. Fast integer multipliers fit in FPGAs (EDN 1993 design idea winner). EDN magazine, May 1994.

[4] F. de Dinechin, J. Detrey, I. Trestian, O. Creţ, and R. Tudoran. When FPGAs are better at floatingpoint than microprocessors. Technical Report ensl00174627, École Normale Supérieure de Lyon, 2007. http://prunel.ccsd.cnrs.fr/ensl-00174627.

[5] F. de Dinechin and V. Lefèvre. Constant multipliers for FPGAs. In Parallel and Distributed Processing Techniques and Applications, pages 167-173, 2000.

[6] A. Dempster and M. Macleod. Constant integer multiplication using minimum adders. Circuits, Devices and Systems, IEE Proceedings, 141(5):407-413, 1994.

[7] J. Detrey and F. de Dinechin. Floating-point trigonometric functions for FPGAs. In Intl Conference on Field-Programmable Logic and Applications, pages 2934. IEEE, Aug. 2007.

[8] J. Detrey, F. de Dinechin, and X. Pujol. Return of the hardware floating-point elementary function. In 18th Symposium on Computer Arithmetic, pages 161-168. IEEE, June 2007.

[9] V. Dimitrov, L. Imbert, and A. Zakaluzny. Multiplication by a constant is sublinear. In 18th Symposium on Computer Arithmetic. IEEE, June 2007.

[10] M. D. Ercegovac and T. Lang. Digital Arithmetic. Morgan Kaufmann, 2003.

[11] A. Y. Khinchin. Continued Fractions. Dover, 1997.

[12] V. Lefèvre. Developments in Reliable Computing, chapter An Algorithm That Computes a Lower Bound on the Distance Between a Segment and $Z^{2}$, pages 203-212. Kluwer Academic Publishers, Dordrecht, 1999.

[13] V. Lefèvre. Multiplication by an integer constant. Technical Report RR1999-06, Laboratoire de l'Informatique du Parallélisme, Lyon, France, 1999.

[14] J.-M. Muller, A. Tisserand, B. D. de Dinechin, and C. Monat. Division by constant for the st100 dsp microprocessor. In 17th Symposium on Computer Arithmetic, pages 124-130, Cape Cod, MA., U.S.A, June 2005. IEEE Computer Society.

[15] Y. Voronenko and M. Püschel. Multiplierless multiple constant multiplication. ACM Trans. Algorithms, 3(2), 2007. 